\title{
A Transparent Robust Quasi-Isotropic Circularly Polarized Antenna for Cub-Sat and Outdoor Wireless
}

\author{
Mansoor Dashti Ardakani ${ }^{1}$ and Marzie Tabatabaefar ${ }^{1}$ \\ ${ }^{1}$ Institut national de la recherche scientifique
}

May 5, 2020

\begin{abstract}
In this paper, a highly robust antenna for omnidirectional circular polarized communication in the harsh environment at $2.45 \mathrm{GHz}$ ISM frequency band is proposed based on a transparent structure. Circular polarization has been realized utilizing a combination of two magnetic and electric dipoles. The antenna is covered with a quarter wavelength layer of plexiglass to achieve desired robustness and visible light transparency. Meanwhile, it can integrate with solar cells because of the high transparency of glass to simultaneously propagate signals and harvest energy. The gain and bandwidth of the antenna are $1.7 \mathrm{dBi}$ and 300 $\mathrm{MHz}$, respectively. The antenna's axial ratio is achieved less than $3 \mathrm{~dB}$ within the bandwidth showing circular polarization behavior. The proposed compact antenna is numerically and experimentally analyzed and compared together, having a suitable agreement. In another aspect, the structure can give promising openings to enhance the propagating properties, which could generate critical advantages for real-world multifunctional applications.
\end{abstract}

\section{Introduction}

Circularly Polarized (CP) antennas can mitigate the multipath interference, propose easier alignment for transmitting and receiving antennas as polarization matching, overcome the phasing issues, and resist the weather conditions. An omnidirectional $\mathrm{CP}$ radiation will result in $360^{\circ}$ coverage desired for multipurpose wireless communications, which is relatively insensitive to transmitter and receiver orientations. Various omnidirectional $\mathrm{CP}$ antennas with conical radiation patterns have been designed over the past years, such as dielectric resonator antenna (DRA) [1], based on tilted dipoles [2], tightly coupled array mechanism [3], multiple-input multiple-output (MIMO) form [4], planar metamaterial structures [5],[6], and fractal formation [7]. Utilizing two electric and magnetic dipoles is a prevalent way to realize orthogonal quadrature fields leading an omnidirectional CP radiation [1],[7]. In order to realize a horizontally polarized magnetic dipole, Alford and Kandoian proposed a loop antenna in the 1950s. Different omnidirectional CP antennas based on the Alford's loop have been proposed in [8],[9].

The use of omnidirectional CP antennas has the advantage that no specific orientations between the transmitter and the load terminal are required for operation. Hence, it can be expected that omnidirectional $\mathrm{CP}$ antennas should find great applications for the WLAN band. For such promising applications, a novel omnidirectional CP antenna with a transparent multi-purpose structure is proposed here. In this letter, a robust omnidirectional $\mathrm{CP}$ antenna for outdoor wireless communication at industrial, scientific and medical (ISM) $2.45 \mathrm{GHz}$ band is presented. The design is developed based on the Alford loop (magnetic dipole) and the electric dipole antennas. Omnidirectional CP, relatively high bandwidth, considering harsh surrounding environments, and multidisciplinary structure for energy harvesting capabilities are the main findings of the reported design. The design has a multifunctional purpose that simultaneously functions as an antenna 
and exploits a possible feature of implementing the solar cell due to the use of transparent material. In the following, the antenna design and its performance are discussed.

\section{Antenna design}

Figure 1 shows the structure of the proposed antenna with the identification of electric and magnetic dipoles. A half wavelength end fed electric dipole is connected to the inner conductor of the common coaxial cable feed. The Alford loop structure is attached to the outer conductor of the coaxial feed, and acts as a magnetic dipole antenna. This magnetic loop antenna behaves electrically as a coil and couples to the magnetic field of the wave, in contrast to the electric dipole antenna which couples to the electric field of the wave. The inner conductor of the feed is a quarter wavelength longer at the center frequency to impose a $90^{\circ}$ phase shift between the electric and magnetic dipoles. The coaxial feed line is placed at the center of the circular structure for the radiation pattern and symmetrical axial ratio (AR).

The two electric and magnetic dipoles are predicted due to the fact that the electric and magnetic vectors are rotating in a circularly polarized antenna. The antenna is covered with a transparent quarter-wavelength layer of plexiglass on the top and bottom to be robustly protected from the harsh environment and as the employment of possible energy saving mechanism. The relative permittivity of plexiglass is calculated about $e_{r}$ $=5.2$ from the microwave interferometry measurement. This method of electromagnetic wave interferometry for calculating the constitutive parameters of a material is reported in [10]. 3D views of the fabricated and simulated versions of the antenna are illustrated in Fig. 2. Each one of the plexiglass layers has a 4 mm height. The metallic parts of the Alford loop are photos plotted on the top of one glass layer with copper material.

As shown in Fig. 2, the antenna is fabricated and tested on a plexiglass substrate to confirm the omnidirectional radiation pattern, $\mathrm{CP}$ property, and the benefit of transparency to the visible light. To geometrically describe the structure in detail, the coordinate axis is shown and the geometrical parameters are given in the caption of Fig. 2 .

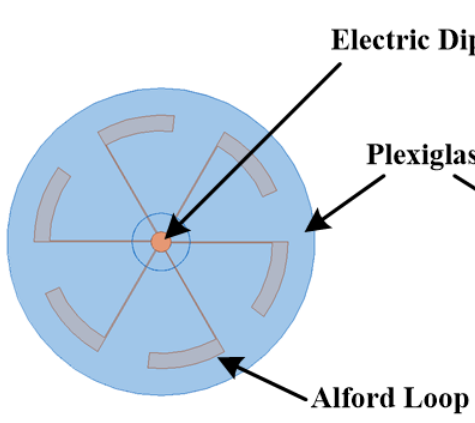

(a)

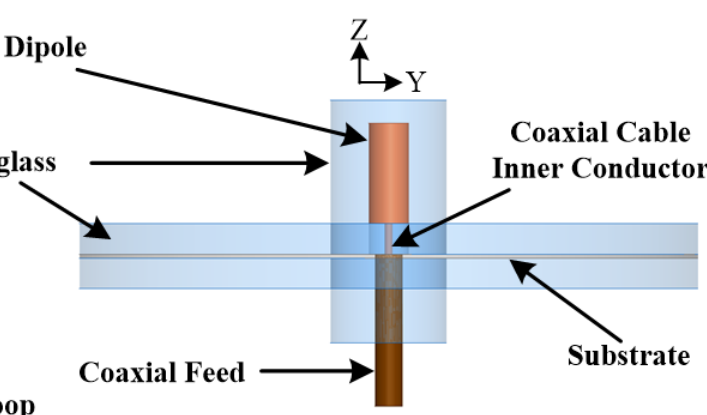

(b)

Layouts of the proposed omnidirectional CP antenna, (a) Top view, and (b) Side view. 


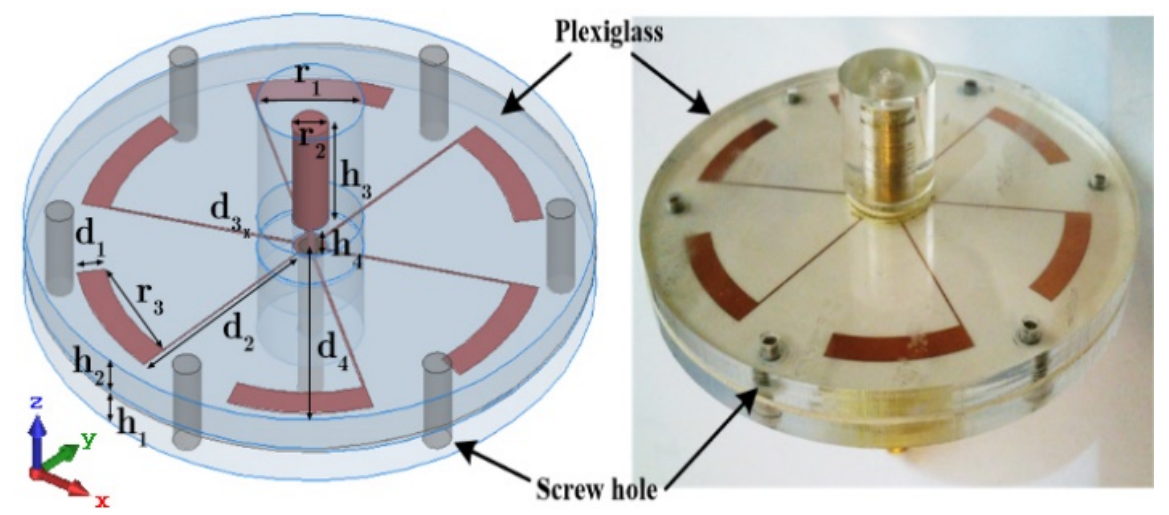

$3 \mathrm{D}$ views of the simulated and fabricated versions of the proposed antenna. Parameters are; $\mathrm{d} 1=4, \mathrm{~d} 2=30.7$, $\mathrm{d} 3=0.3, \mathrm{~d} 4=40, \mathrm{~h} 1=4, \mathrm{~h} 2=4, \mathrm{~h} 3=13, \mathrm{~h} 4=4, \mathrm{r} 1=15, \mathrm{r} 2=5.2$, and $\mathrm{r} 3=17.92$ (unit: $\mathrm{mm}$ ).

\section{Results and discussions}

Given the Fig. $3 a$, the scattering curves are obtained from full-wave simulation software and compared with measurement to analyze the antenna parameter. The reflection coefficient as the impedance bandwidth $\left(\left|\mathrm{S}_{11}\right|<-10 \mathrm{~dB}\right)$ shows the operating frequency of the antenna starts from 2.35 to $2.8 \mathrm{GHz}$, corresponding to a fractional bandwidth of $0.98 \%$. In order to have a $\mathrm{CP}$ antenna, the AR of the antenna must be less than $3 \mathrm{~dB}$. The AR of the proposed antenna is achieved less than $3 \mathrm{~dB}$ in the $2.4-2.7 \mathrm{GHz}$ frequency band according to Fig. $3 b$. Hence, the achieved AR is within the 10-dB bandwidth. The simulated and measured results are presented in Fig. 3, in which the blue dashed line is from measurement while the red line represents the results from the CST software. In addition, Fig. 4 shows the AR of the antenna in $x y$ -plane versus $\varphi$ angle. It is less than $3 \mathrm{~dB}$, thus it owns a proper omnidirectional CP radiation. The three charts are from three different frequencies of $2.4,2.5$, and $2.6 \mathrm{GHz}$. These results prove that the designed antenna radiates $\mathrm{CP}$ waves.

The proposed design is located far enough from the antenna measurements inside the anechoic chamber in the far-field region to guarantee plane wave impingement. E- and H-plane radiation patterns of the studied antenna are presented in Fig. 5. Simulations and measurements show a quasi-perfect omnidirectional radiation. From the results, a stable antenna gain of $1.7 \mathrm{dBi}$ is indicated. The solid red chart represents simulation, and the dashed blue is the result of measurement as given in the Fig. 5. The attained results corroborate with each other. The 3D directivity of the antenna is shown and plotted in Fig. 6 that a good omnidirectional pattern is observed. The $\mathrm{CP}$ radiation pattern is identical to the whole $x y$-plane. 


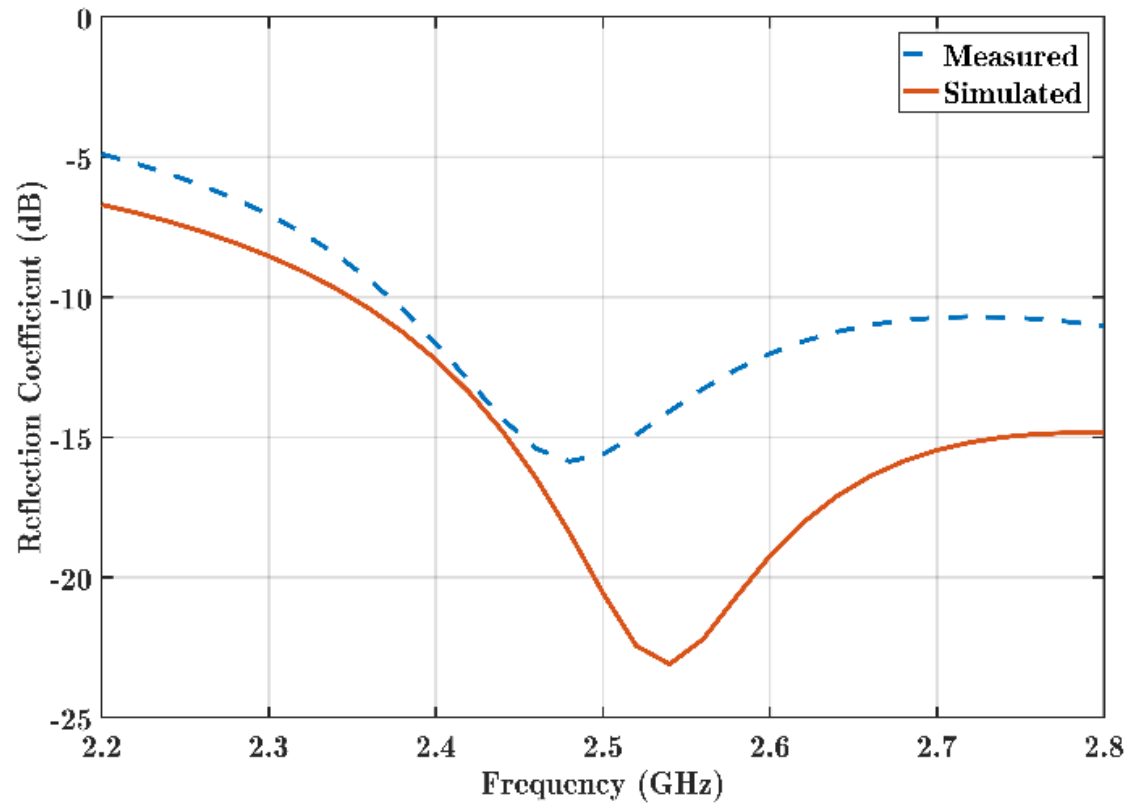

(a)

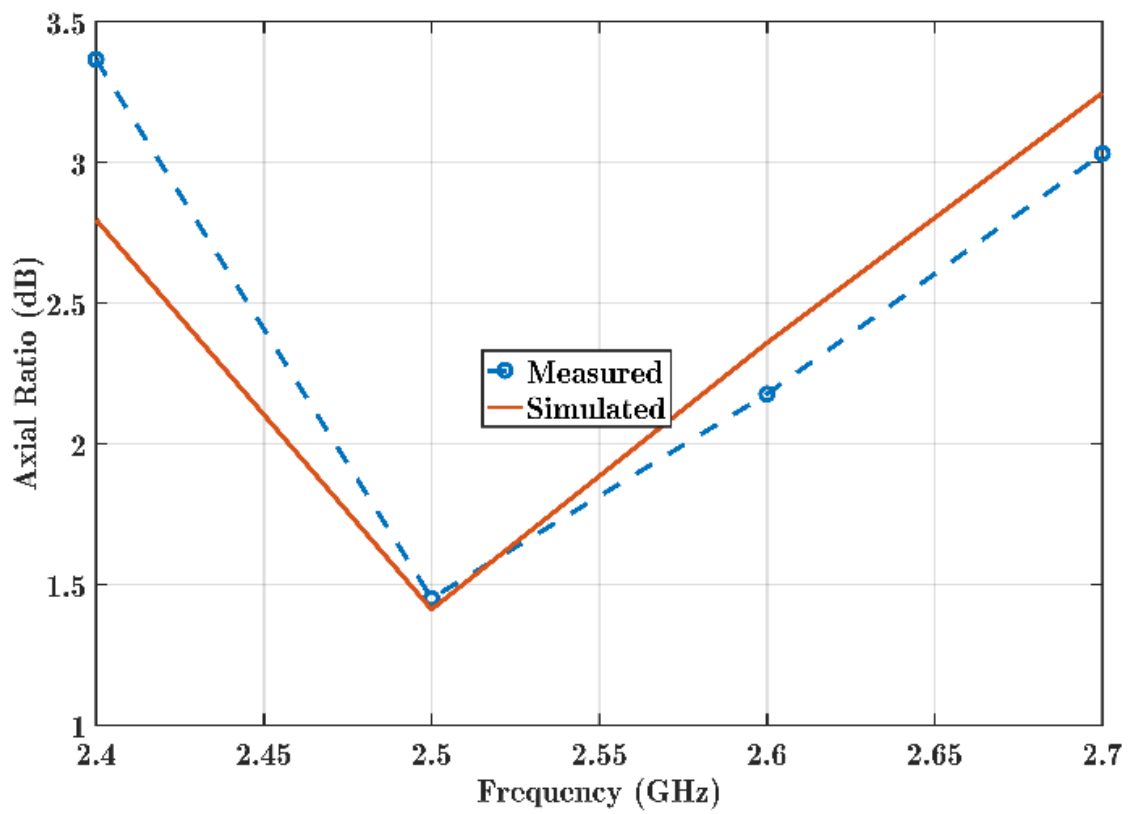

(b)

Measured and simulated results for; (a) reflection coefficient $\left(S_{11}\right)$, and (b) axial ratio (AR) of the proposed antenna versus frequency (at $\vartheta=90^{\circ}$ ). 


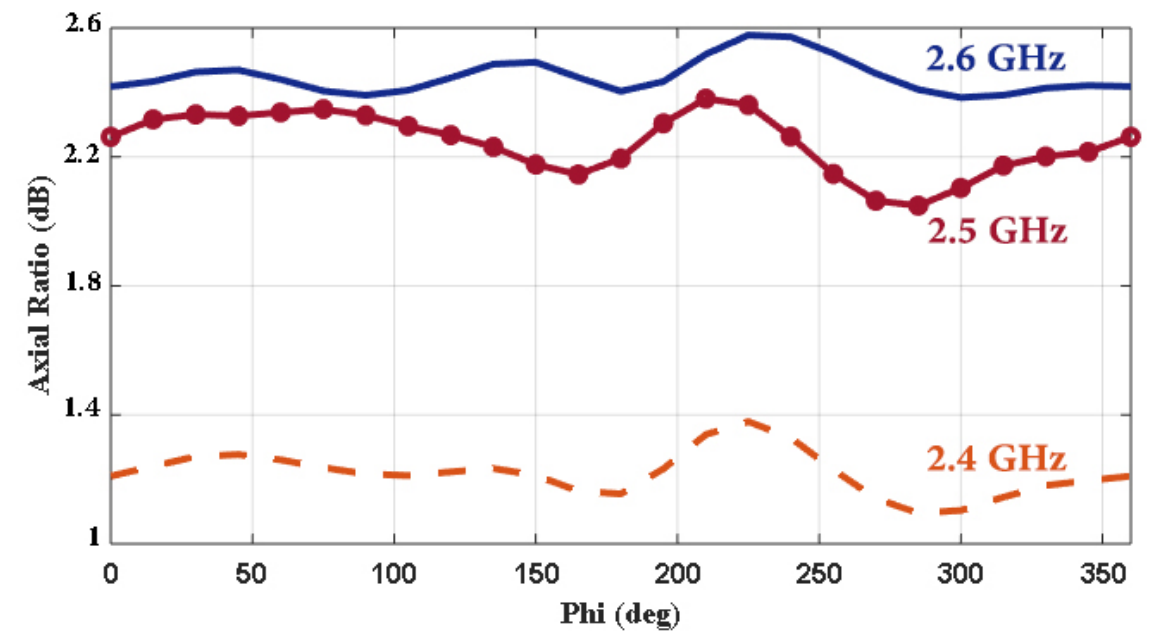

AR of the proposed antenna in xy-plane (against the $\varphi$ angle) for three frequencies from simulation software.

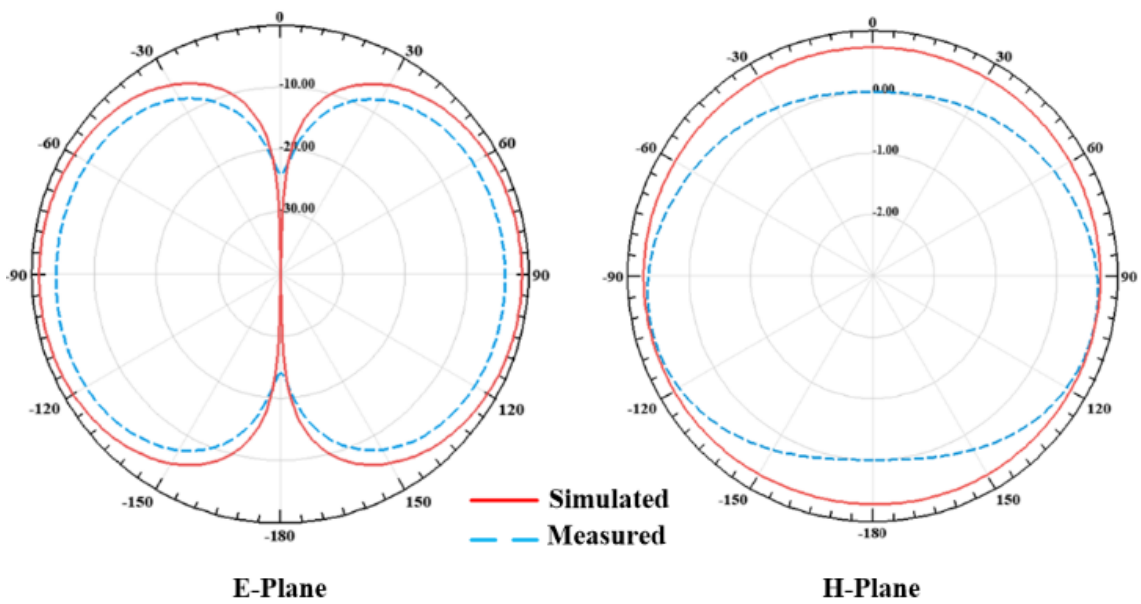

E- and H-plane directivity of the proposed antenna at $2.45 \mathrm{GHz}$. 

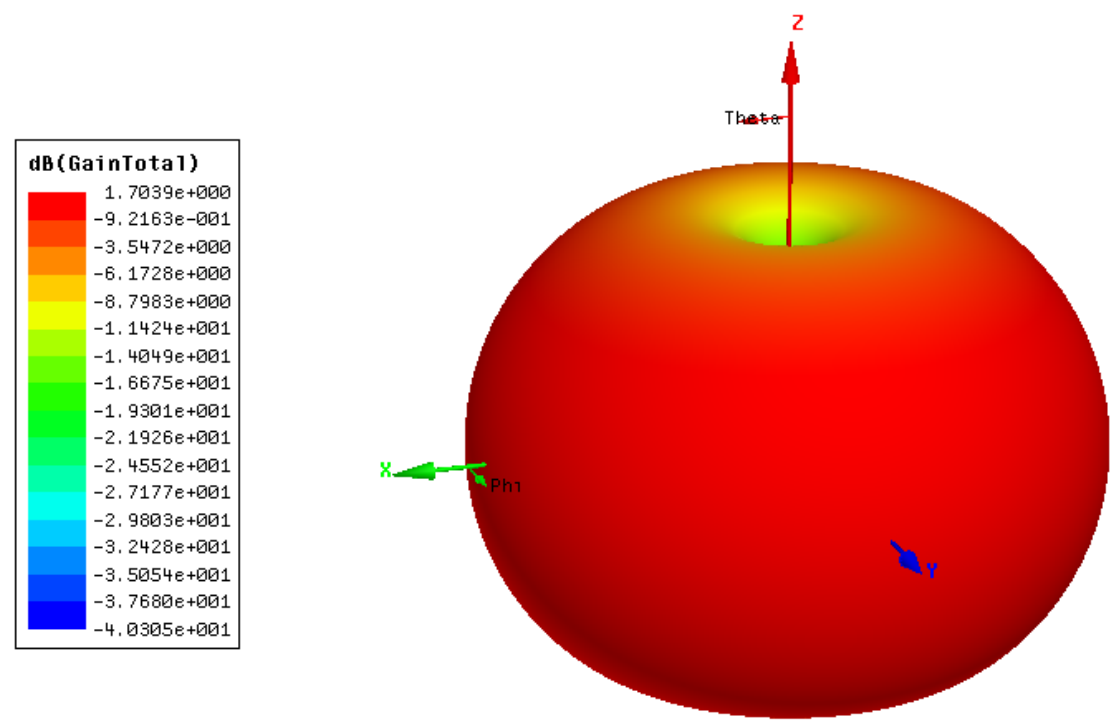

$3 \mathrm{D}$ radiation pattern of the proposed antenna at $2.45 \mathrm{GHz}$.

\section{Conclusion}

A new approach of multifunctional robust omnidirectional CP antenna for outdoor wireless communication at $2.45 \mathrm{GHz}$ ISM band has been proposed using transparent material. The CP is realized utilizing two electric and magnetic dipole structures based on the plexiglass material covering. The discussed curved branches present a magnetic dipole situation. The antenna is covered with layers of plexiglass for the robustness and potential capability of integrating with the solar cells as an energy harvesting mechanism. Therefore, the proposed antenna can act as a CP omnidirectional antenna and energy harvesting device simultaneously. The peak radiation gain of the antenna is achieved about $1.7 \mathrm{dBi}$ for $\mathrm{CP}$. The axial ratio of the antenna from 2.4 to $2.7 \mathrm{GHz}$ is measured less than $3 \mathrm{~dB}$. The radiation pattern of the antenna shows a perfect symmetric omnidirectional pattern around its axis in the azimuthal $x y$-plane. Due to multidisciplinary and multifunction capacities, the proposed antenna has potential applications in cube-sate and flying object devices.

\section{References}

1. W. Li, and K. W. Leung, "Omnidirectional Circularly Polarized Dielectric Resonator Antenna With Top-Loaded Alford Loop for Pattern Diversity Design," IEEE Transactions on Antennas and Propagation, vol. 61, no. 8, pp. 4246-4256, Aug. 2013.

2. Y. Fan, X. Quan, Y. Pan, Y. Cui, and R. Li, "Wideband Omnidirectional Circularly Polarized Antenna Based on Tilted Dipoles," in IEEE Transactions on Antennas and Propagation, vol. 63, no. 12, pp. 5961-5966, Dec. 2015.

3. Y. Liu, H. Liu, and S. Gong, "Wideband polarization-reconfigurable antenna based on tightly coupled array mechanism," 12th European Conference on Antennas and Propagation (EuCAP 2018), London, 2018, pp. 1-3.

4. C. H. Ahn, S. W. Oh, and K. Chang, "A dual-frequency omnidirectional antenna for polarization diversity of MIMO and wireless communication applications," IEEE Antennas Wireless Propag. Lett., vol. 8, pp. 966-969, 2009.

5. I. W. Cao, A. Liu, B. Zhang, T. Yu, and Z. Qian, "Dual-Band Spiral Patch-Slot Antenna With Omnidirectional CP and Unidirectional CP Properties," IEEE Transactions on Antennas and Propagation, 
vol. 61, no. 4, pp. 2286-2289, April 2013.

6. M. Dashti Ardakani, and R. Amiri, "Mutual Coupling Reduction of Closely Spaced MIMO Antenna Using Frequency Selective Surface based on Metamaterials", The Applied Computational Electromagnetics Society (ACES) Journal, vol. 32, No. 12, Dec. 2017.

7. J. S. Row, and M. C. Chan, "Reconfigurable Circularly-Polarized Patch Antenna With Conical Beam," IEEE Transactions on Antennas and Propagation, vol. 58, no. 8, pp. 2753-2757, Aug. 2010.

8. B. C. Park, and J. H. Lee, "Omnidirectional Circularly Polarized Antenna Utilizing Zeroth-Order Resonance of Epsilon Negative Transmission Line," IEEE Transactions on Antennas and Propagation, vol. 59, no. 7, pp. 2717-2721, July 2011.

9. B. C. Park, and J. H. Lee, "Dual-Band Omnidirectional Circularly Polarized Antenna Using Zerothand First-Order Modes," IEEE Antennas and Wireless Propagation Letters, vol. 11, pp. 407-410, 2012.

10. B. Chaudhury, and S. Chaturvedi, "Study and optimization of plasmabased radar cross section reduction using three-dimensional computations," IEEE Trans. Plasma Sci., vol. 37, no. 11, pp. 2116-2127, Nov. 2009. 